\title{
Comparative Evaluation of Effectiveness of Tell-Play-Do, Film Modeling and Use of Smartphone Dental Application in the Management of Child Behavior
}

\author{
Mital V Kevadia ${ }^{1}$, Sandhyarani B ${ }^{2}$, Anil T Patil ${ }^{3}$, Sachin A Gunda ${ }^{4}$
}

\begin{abstract}
Aim: To evaluate the effectiveness of three different behavioral modification techniques: tell-play-do (TPD), film modeling, and use of smartphone dental app in the management of child behavior.

Settings and design: Seventy-five children aged between 6 years and 9 years who were on their first dental visit were randomly divided into three groups. The study was conducted into two visits.

Materials and methods: First visit: group I—children watched a film containing various dental procedures performed on a child model followed by oral prophylaxis; group II-TPD techniques were introduced with dental instruments imitating various playing objects followed by oral prophylaxis; group III-children were asked to use mobile dental application followed by oral prophylaxis. Second visit: After 7 days interval, all the children were subjected to class I restorative treatment using glass-ionomer cement. In both visits, the heart rate (HR), facial image scale (FIS) score, and Venham's pictorial index (VPI) score were evaluated before the intervention, after the intervention of behavior modification technique, and during the dental procedure.

Statistical analysis used: One-way ANOVA test followed by the post hoc Tukey test was used to compare HR and the Kruskal-Wallis test was used to compare the FIS and VPI of all three groups.

Results: The average HR, FIS, and VPI scores were significantly lower among children who received TPD intervention compared to those who received film modeling intervention and mobile dental application.

Conclusion:Tell-play-do technique is more effective in reducing children's fear and anxiety for dental treatment. Tell-play-do can be a functional alternative method to tell-show-do (TSD) and modeling techniques during dental treatment.

Keywords: Behavior modification, Dental anxiety, Film modeling, Smartphone app, Tell-play-do.

International Journal of Clinical Pediatric Dentistry (2020): 10.5005/jp-journals-10005-1857
\end{abstract}

\section{INTRODUCTION}

The primary emotion of a child while entering into a dental office is anxiety and fear. ${ }^{1}$ Dental anxiety and fear being attributed by many as one of the major causes to avoid seeking dental care by children and is one of the big challenges in the dental office. ${ }^{1}$ Alleviating a child's dental anxiety is needed in mitigating the immediate fear and it also prevents apprehension from continuing in the later stages of life. It is of vital importance that any approach to behavioral management for the dental child patient must be implanted in empathy and a concern for the well-being of every child. ${ }^{2}$

The American Academy of Pediatric Dentistry (AAPD) recommended concentrating more on non-pharmacologic intervention in future studies. ${ }^{3}$ Most commonly used behavior management techniques (BMTs) by pediatric dentists are tellshow-do (TSD) and modeling. ${ }^{2,4-8}$

Several studies have evaluated the efficiency of film modeling in the reduction of a child's dental anxiety. ${ }^{9-12}$ In film modeling, children were prepared for dental procedures by observing a film in which various dental procedures were performed on a child model having cooperative behavior. Children may reproduce behavior exhibited by the child model in the film.

However, just by observing a model, explaining, or demonstrating the dental procedure may not provide a more explanatory concept, instead of it makes them play with dental imitating instrument toys which will provide a more explanatory
${ }^{1-4}$ Department of Pedodontics and Preventive Dentistry, Bharati Vidyapeeth Dental College, Sangli, Maharashtra, India

Corresponding Author: Mital V Kevadia, Department of Pedodontics and Preventive Dentistry, Bharati Vidyapeeth Dental College, Sangli, Maharashtra, India, Phone: +919503003914, e-mail: mitalkevadia@ gmail.com

How to cite this article: Kevadia MV, B Sandhyarani, Patil AT, et al. Comparative Evaluation of Effectiveness of Tell-Play-Do, Film Modeling and Use of Smartphone Dental Application in the Management of Child Behavior. Int J Clin Pediatr Dent 2020;13(6):682-687.

Source of support: Nil

Conflict of interest: None

concept. Using the idea of learning by doing concept TSD technique was modified to tell-play-do (TPD). In this, children were asked to play with dental imitating toys and explained about it which includes various diagnostic instruments (mouth mirror and dental probe), airotor, suction (saliva ejector), and air/ water syringe.

Nowadays children from all age groups play countless games on the phone, talking to their friends for long hours, or even browsing the internet. Today, we have many apps available on mobile phones to educate the children about the procedures of their dental treatment which can help to alleviate fear. 
On search of the literature, a small number of studies could be found on the management of pediatric patients using the TPD technique and dental apps.

This study aimed to evaluate the effectiveness of three different behavioral modification techniques; TPD, film modeling, and the use of smartphone dental app in the management of child behavior during dental practice.

\section{Materials and Methods}

After obtaining the ethical clearance from the institutional review board and written consent from parents, 75 children having age between 6 year and 9 years ( \pm 4 months) of age were enrolled in the study based on inclusion and exclusion criteria.

\section{Inclusion Criteria}

Children aged between 6 years and 9 years having a first dental visit and no previous history of hospitalization. A child should have a caries cavity lesion in one of the primary molars and needed class I glass-ionomer restoration without local anesthesia was included in the study.

\section{Exclusion Criteria}

Children who were from single-parent families and mentally challenged that could compromise their understanding of the study, those who were undergoing medical treatment that might affect heart rate $(\mathrm{HR})$, and those with heartbeat disorders were excluded from the trial.

Each child's parents or guardians were explained in detail about the study. They were informed about their right to refuse or discontinue their participation at any time and were then asked to sign the consent form.

\section{Study Design}

Study subjects were randomly allocated into three groups:

- Group I: Children who were prepared for dental treatment by the film modeling technique.

- Group II: Children who were prepared for dental treatment by the TPD technique.

- Group III: Children who were prepared for dental treatment by using a mobile dental app called "My Little Dentist" developed by Tenolgix Games available on the Google Play Store on smartphones.

The study was divided into two visits.

\section{First Visit}

\section{Group I}

In group I, the child was directed toward the room to watch a film (Fig. 1). Children watched a film containing various dental procedures like TSD, prophylaxis, and restorative procedure performed on a 6-year-old cooperative child model for 15 minutes followed by oral prophylaxis. (The produced film had been approved by three pediatric dentists.) The duration of this was standardized for 15 minutes and then the child was directed to the clinical area and oral prophylaxis was performed.

\section{Group II}

In group II, the child was taken to the play area, where the customized dental imitating instrument toys and a cartoon character (Chotta Bheem) with mouth wide open were available.

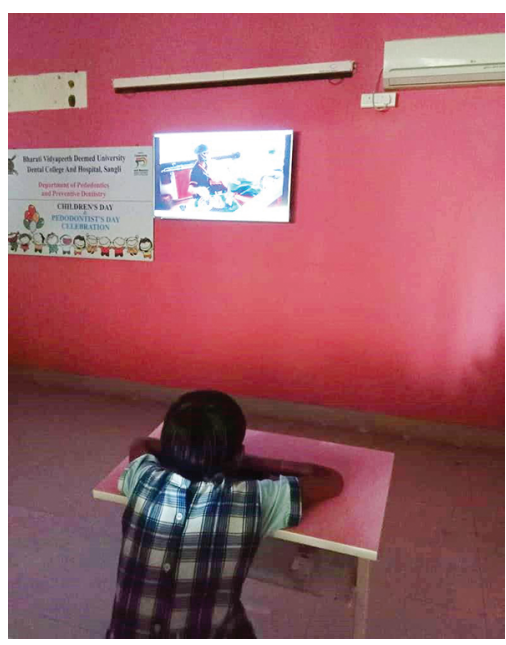

Fig. 1: Film modeling

The child was explained about all the dental imitating instrument objects using appropriate euphemisms and procedures in phrases appropriate to the developmental level of the child and they were allowed to play with dental imitating instrument toys including diagnostic instruments (mouth mirror and dental probe), airotor, suction (saliva ejector), air/water syringe tips to play and perform a dental procedure on the cartoon character (Fig. 2). Also, the noise was incorporated in the dental object resembling a clinical sound effect followed by oral prophylaxis. The duration of this was standardized for 15 minutes; then, the child was directed to the clinical area and oral prophylaxis was performed.

\section{Group III}

The child was made to make use of the "My Little Dentist" app and perform virtual dental treatments (Fig. 3). The app gave an idea to the child how and what would be the nature of their treatment. The children were virtually made dentists. They were made to perform dental procedures that included oral prophylaxis, filling, and extractions. The duration of this was standardized for 15 minutes; then, the child was directed to the clinical area and oral prophylaxis was performed.

\section{Evaluation at the First Visit}

Each group's children were evaluated in the following sequence:

\section{Before Intervention}

Immediately after the child entered the department check anxiety level by measuring HR (using Gibson finger oximeter), facial image scale (FIS), and Venham's pictorial scale (VPS).

\section{After Intervention}

The respective child was directed to a separate room to receive a particular intervention like Filmed modeling or TPD or allowed to use a dental app. Then, again, all parameters were noted.

\section{During Oral Prophylaxis}

During the oral prophylaxis procedure, all parameters were noted.

\section{Second Visit}

After 7 days interval, all the children were subjected to restorative treatment. In this visit, the occlusal cavity (class I) was prepared for the restoration of teeth using glass-ionomer cement using the same 


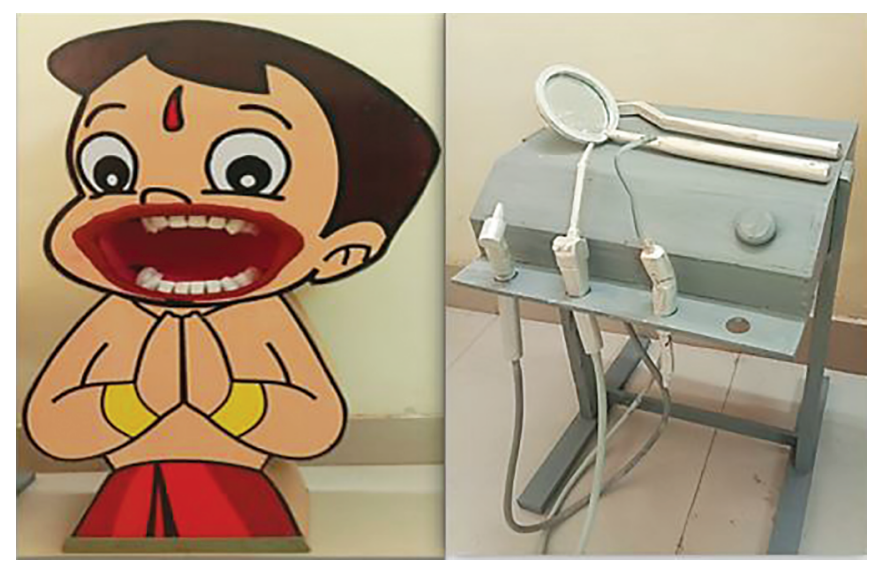

Fig. 2: Tell-play-do model (Chotta Bheem) and dental instrument imitating toys

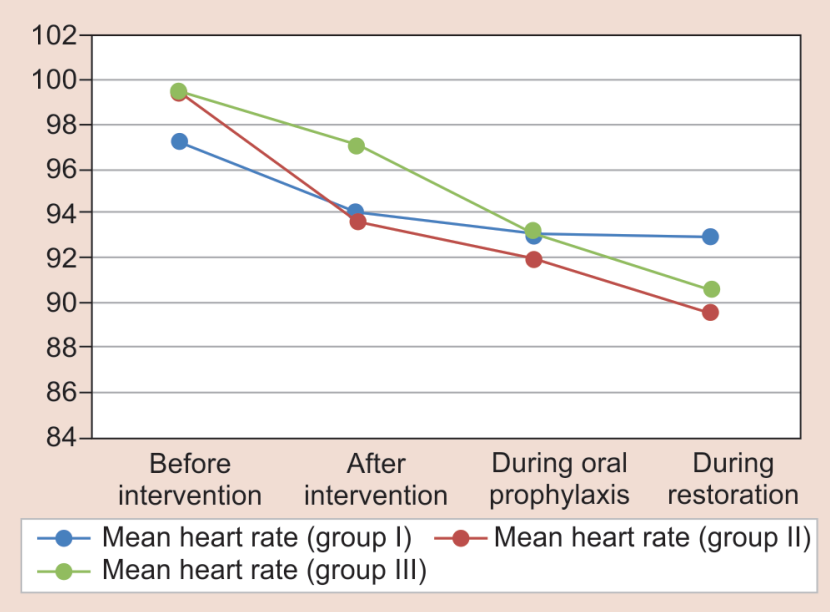

Fig. 4: Comparison of change in HR among three groups

treatment protocol. The duration of the whole procedure was 30 minutes for each child.

\section{Evaluation at Second Visit}

The child's response was noted before and during cavity preparation and restoration. Check for HR (using Gibson finger oximeter), FIS, and VPS.

\section{Statistical Analysis}

Data obtained were compiled on an MS Office Excel Sheet and were subjected to statistical analysis using the Statistical Package for Social Sciences (SPSS v 21.0, IBM).

Mean HR (numerical value) was compared in-between the three groups using a one-way ANOVA test. Change of HR at different duration was compared in-between three groups using the post hoc Tukey test. Kruskal-Wallis test was used to compare the FIS and Venham's pictorial index (VPI) of all the groups depending on their mean ranks. For all the statistical tests, $p<0.05$ was considered to be statistically significant.

\section{Results}

A total number of 75 children participated in the study and were allocated between three groups, i.e., film modeling $(n=25)$, TPD

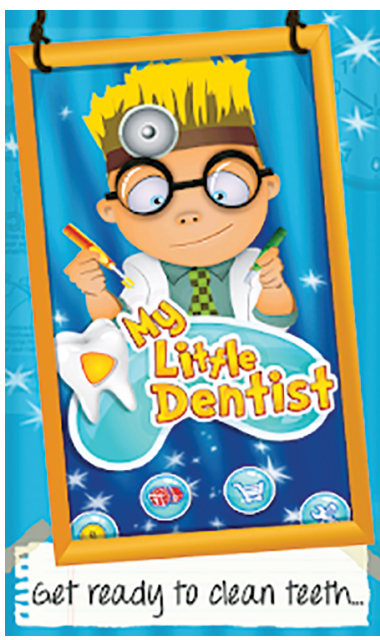

Fig. 3: The dental app (“My Little Dentist" app)

$(n=25)$, and smartphone dental application $(n=25)$. Data revealed that all three groups were the same in demographic characteristics including their mean age and participation in the same school.

Oral screening, oral prophylaxis, and restorative treatment were completed for each group. The data obtained were statistically analyzed using SPSS version 21.0.

Mean HRs were significantly lower among children in group II than among those in group I during the first visit (after intervention), $p=0.029$, and also in the second visit (during restoration), $p=$ 0.033 . Similarly, the mean change in HRs was significantly lower in group II when compared with that of group III children (after intervention), $p=0.046$, and in group I when compared with group III (after intervention), $p=0.031$, at the first visit. Also, the mean HR was significantly lower in-between group II and group III (during restoration), $p=0.050$.

Accordingly, HR mean scores during the first visit (before the intervention and during the oral prophylaxis procedure) showed no significant differences $p=0.349$ and $p=0.853$, respectively. Overall percentage reduction in HR was more in group II (12.64\%) than in group I (11.83\%) and group III (4.40\%) in the second visit (after restoration). Mean HR at different intervals was significantly lower among children in group II than among those in group I and group III (Fig. 4).

The difference in FIS scale score was significantly lower in-between group II when compared with group I and group III in the first visit (after intervention) $p=0.029$ and $p=0.031$, respectively. Similarly, the mean change in the FIS scale score was significantly lower in group I when compared with that of group III children in the first visit (after intervention) $p=0.046$. Mean FIS scores before intervention, during oral prophylaxis, and during procedure (first visit) showed no significant differences $p=0.477, p=0.539$, and $p=0.664$, respectively. Overall unit reduction in FIS was more in group II (1.76\%) than in group I (1.52\%) and group III (1.36\%) in the second visit (after restoration). Mean FIS at different intervals was significantly lower among children in group II than among those in group I and group III (Fig. 5).

The mean VPI scale score was significantly lower in-between group II when compared with group I and group III (after intervention) $p=0.040$ and $p=0.045$, respectively. Similarly, the mean change in VPI score was significantly lower in group II when compared with that of group III children in the second 


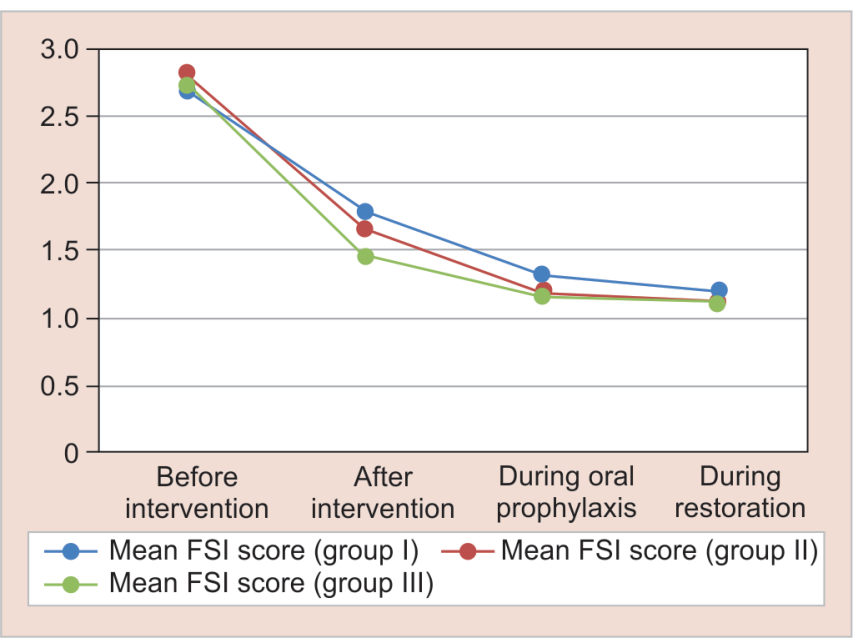

Fig. 5: Comparison of change in FIS among three groups

visit (during restoration) $p=0.025$. Accordingly, mean VPI scores before the intervention and during oral prophylaxis procedure (first visit) showed no significant differences $p=0.377$ and $p=$ 0.173 , respectively. Mean VPI at different intervals was significantly lower among children in group II than among those in group I and group III (Fig. 6).

\section{Discussion}

Dental fear is a common problem in children and adolescents. Fear of the unknown provokes anxiety in the dental office. It is one of the major causes of avoid seeking dental care for children. ${ }^{13}$ The child's behavior on dental visits depends on various variables like age, past medical and dental history, parent's anxiety for seeking dental treatments, and parental behavior., ${ }^{43}$

The management of children's behavior is a vital component of pediatric dental practice. The first dental visit plays an inherent role in eliciting a positive dental attitude. Cooperation of child during dental treatment is of prime importance to render good quality of treatment. ${ }^{5}$

Behavior management techniques are aimed at enhancing a child's cooperation, establish proper communication, decrease fear and anxiety, render successful and good quality dental care, build a trusting relationship between the dentist, child, and parent, and promote the child's positive dental attitude.

To date, many BMTs are available to dental practitioners, namely, TSD, desensitization, modeling, distraction, positive reinforcement, hand-over-mouth, voice control, restrain/protective stabilization, conscious sedation, and general anesthesia. However, TSD and modeling technique are the foundation of the child's education and behavior guidance at first visits. Modeling can be either live or film modeling. Tell-show-do and modeling depend on learning theory in which children are either explained or demonstrated about the dental procedure. However, the TPD technique is based on learning by doing theory. It may give more relevant information to children about various dental instruments and dental procedures and can be a more practical way to understand the dentist frame of reference. .,5,13 $^{4}$

Nowadays children use mobile phones for various purposes like playing games, communicating with friends, or browsing the internet for fun. Hence, the mobile phone can be used for patient

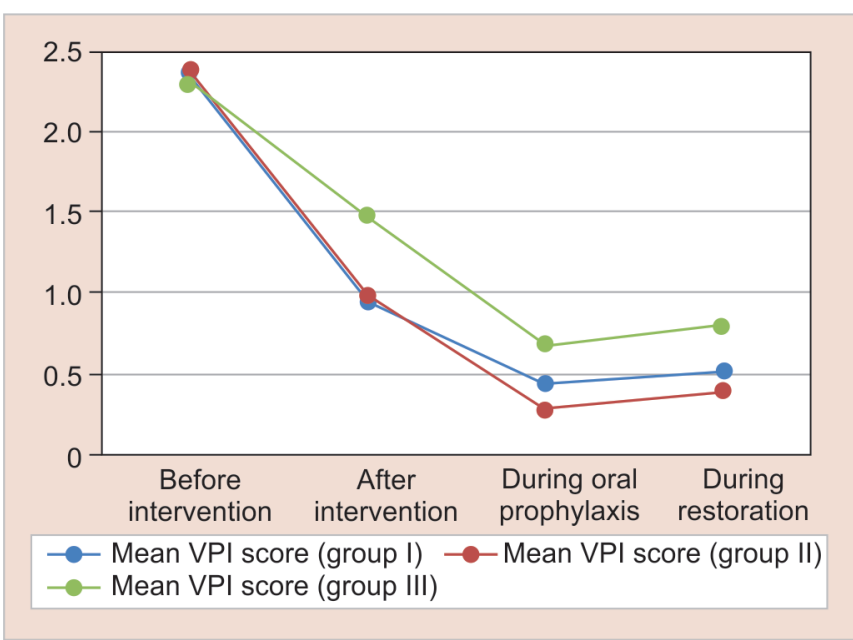

Fig. 6: Comparison of change in VPI among three groups

education. There are many dental apps available that give clinical information to children in a way that is easy to understand. It provides 3D images, different treatment plans, and educational counseling, so the patients can better understand their course of treatment.

This study was designed to evaluate and compare the efficiency of film modeling, TPD technique, and the use of smartphone dental application in reducing child anxiety during dental treatment.

In the TPD technique, a two-way interchange of information takes place between dentist and child. After performing dental treatment by using dental imitating toys, the child gets an idea about the various dental procedures. Also, he gets exposed to various sights and noise of equipment used in dental practice. This develops more cooperative behavior. ${ }^{5}$

Vishwakarma et al. in 2017 have compared two different behavioral modification techniques; TPD and live modeling among 5- to 7-year-old children and reported that the TPD technique is more efficient than the live modeling technique to reduce children's fear and achieve more cooperative behavior during treatment. They found that mean HR at different intervals was significantly lower among children in the TPD group than among those in the live modeling group during the first visit (after the intervention and during the procedure) and also in the second visit (during the procedure). Hence, the TPD technique may be an alternate method to TSD and live modeling technique. ${ }^{5}$

Patil et al. in 2017 have conducted a study on 60 children who were made to use a mobile dental app called "My Little Dentist" which is developed by Tenlogix Games available on the Google Play Store on smartphones. Their anxiety levels were noted before and after playing the game using the face imaging scale. The results were found to be highly significant; $86.67 \%$ of patients turned from a negative to positive behavior, $11.67 \%$ from positive to definitely positive, and $1.67 \%$ from definitely negative to negative according to Frankl's behavior rating scale and reported that the mobile dental app is useful to alleviate child's fear and anxiety toward dental treatment and it can be used as an adjunct with the conventional behavior management techniques. ${ }^{6}$

Sharma and Tyagi in 2011 have conducted a retrospective study and assessed 328 children's behavior using Frankl's behavior rating scale during dental visits. Live modeling and TSD behavior management techniques were used before conducting any 
procedure. They reported that proper assessment of children's behavior can help the dentist to use proper treatment plan by using more appropriate behavior management techniques and both, live modeling and TSD are very effective in modifying a child's behavior. ${ }^{2}$

The study results of and Farhat-McHayleh et al. in $2009^{7}$ and Alrshah et al. $2014^{8}$ showed that children's HRs were significantly less when the mother was available as a live model as compared to father. Also, live modeling is more effective as compared to the TSD technique to reduce a child's anxiety and fear.

Paryab and Arab in 2014 evaluated the effect of filmed modeling in comparison with TSD technique children aged between 4 and 6 years during dental practice. In both groups, HR and behaviors of children using Venham and Frankl rating scales were recorded and found a statistically significant difference between both the groups. They reported that filmed modeling can be an alternative method to the TSD technique. ${ }^{14}$

Facial image scale and VS were used to quantify the Child's behavior reactions as these are the easiest and one of the fastest methods with reliability and validity for statistical analysis. ${ }^{15}$ In this study, analysis of FIS and VS revealed that the fear perception range by group II (TPD) was significantly lower compared to group I (live modeling) and group III (mobile dental application).

Humphris et al. in 1995 have used a similar scale (FIS and VS) for study and reported that live modeling was a more effective technique than TSD in reducing a child's anxiety. ${ }^{16}$

In the present study, modification of TSD to TPD is significantly effective than film modeling in reducing not only the HR (physiological index) but also the cooperative behavior (FIS and VS). Also, the use of the mobile dental application in educating patients was less effective as compared to TPD to reduce the child's anxiety.

Also, the number of children showing more positive behavior had increased after the intervention of behavior modification technique in all three groups.

Before intervention to after intervention, the number of children showing positive response and cooperative behavior was increased more in the TPD technique (group I) followed by film modeling (group I) and then in the subjects who were subjected to play with the mobile dental application (group I). From the first visit to the second visit, the number of children showing more cooperative behavior was significantly more in group II than in group I and group III.

Similar studies conducted by Howitt and Stricker in $1970^{17}$ and Sharma and Tyagi in $2011^{2}$ also concluded that a child's arousal level was reduced as they became accustomed to the dental situation.

According to our study, the TPD technique among 6- to 9-yearold children is impressively effective and $87 \%$ were definitely positive during the second visit of the restorative procedure.

The results of this study showed that the TPD technique was more effective among all the three groups on child anxiety levels and increased the cooperative behavior during dental treatment in children aged between 6 years and 9 years.

By simple modification of TSD to TPD can have a good impact on younger children, so that they can feel comfortable and accept the dental treatment. Ultimately, it brings about more cooperative behavior. However, children should be continuously under observation while using mobile phones. Also, in the present study, a fewer number of patients were included and patients with past negative dental/medical history were excluded. Hence, more studies are required for evaluating the success rate of TPD and the use of smartphone apps for patient education.

\section{Conclusion}

According to the methodology and the results of the present study, We can conclude that:

- Tell-play-do technique is more efficient than film modeling technique and use of the mobile dental application to control 6-9-year-old children's anxiety to achieve more cooperative behavior during dental treatment as TPD technique gives more conceptual insight about the dental instruments and concrete experience to dental procedures to children before starting any treatment.

- Use of mobile dental applications can be used as an adjunct with conventional behavior modification techniques.

\section{Clinical significance}

Tell-play-do technique can be used as an alternative method to TSD and film modeling technique for managing a child's anxious behavior.

\section{References}

1. Sharath A, Rekka P, Muthu MS, et al. Children's behavior pattern and behavior management techniques used in a structured postgraduate dental program. J Indian Soc Pedodont Prevent Dentis 2009;27(1):22. DOI: 10.4103/0970-4388.50812.

2. Sharma A, Tyagi R. Behavioural assessment of children in dental settings: a retrospective study. Int J Clin Pediatr Dent 2011;4(1):35-39. DOI: 10.5005/jp-journals-10005-1078.

3. American Academy on Pediatric Dentistry Clinical Affairs CommitteeBehavior Management Subcommittee, American Academy on Pediatric Dentistry Council on Clinical Affairs. . Guideline on behavior guidance for the pediatric dental patient. Pediatr Dent 2008;30(7 Suppl):125.

4. Kuhn BR, Allen KD. Expanding child behavior management technology in pediatric dentistry: a behavioral science perspective. Pediatr Dent 1994;16:13.

5. Vishwakarma AP, Bondarde PA, Patil SB, et al. Effectiveness of two different behavioral modification techniques among 5-7-year-old children: a randomized controlled trial. J Indian Soc Pedod Prev Dent 2017;35(2):143-149. DOI: 10.4103/JISPPD.JISPPD_257_16.

6. Patil VH, Vaid K, Gokhale NS, et al. Evaluation of effectiveness of dental apps in management of child behaviour: a pilot study. Int J Pedod Rehabil 2017;2(1):14-18. DOI: 10.4103/ijpr.ijpr_5_17.

7. Farhat-McHayleh N, Harfouche A, Souaid P. Techniques for managing behaviour in pediatric dentistry: comparative study of live modeling and tell-show-do based on children's heart rates during treatment. J Can Dent Assoc 2009;75:283.

8. Alrshah SA, Kalla IH, Abdellatif AM. Live modeling vs. tell-show-do technique for behaviour management of children in the first dental visit. Mansoura J Dent 2014;1:72-77.

9. Melamed BG, Hawes RR, Heiby E, et al. Use of filmed modeling to reduce uncooperative behaviour of children during dental treatment. J Dent Res 1975;54(4):797-801. DOI: 10.1177/00220345750540041701.

10. Fields $\mathrm{H}$, Pinkham J. Videotape modeling of the child dental patient J Dent Res 1976;55(6):958-963. DOI: 10.1177/00220345760550064601.

11. Melamed BG, Yurcheson R, Flees EL, et al. Effects of film modeling on reduction of anxiety-related behaviours in individuals varying in level of previous experience in the stress situation. J Consult Clin Psychol 1978;46(6):1357-1367. DOI: 10.1037/0022-006X.46.6.1357. 
12. Rouleau J, Ladouceur R, Dufour L. Pre-exposure to the first dental treatment. J Dent Res 1981;60(1):30-34. DOI: 10.1177/00220345810600010601.

13. Cianetti S, Lombardo G, Lupatelli E, et al. Dental fear/anxiety among children and adolescents. A systematic review. Source Eur J Paediat Dentis 2017;18(2):121-130. Local Messages issues held at the bda library from 2002 onwards, to request this article from the library go to. European Journal of Paediatric Dentistry, 18(2), pp. 121-130.

14. Paryab $M$, Arab Z. The effect of filmed modeling on the anxious and cooperative behavior of 4-6 years old children during dental treatment: a randomized clinical trial study. Dent Res J 2014;11(4): 502.

15. Allen KD, Stanley RT, McPherson K. Evaluation of behavior management technology dissemination in pediatric dentistry. Pediatr Dent 1990;12:79-82.

16. Humphris GM, Morrison T, Lindsay SJ. The modified dental anxiety scale: validation and United Kingdom norms. Community Dent Health 1995;12:143-150.

17. Howitt JW, Stricker G. Sequential changes in response to dental procedures. J Dent Res 1970;49(5):1074-1077. DOI: 10.1177/00220345700490051201. 